\title{
Development of a device for detecting target specimens from EUS-guided FNA samples
}

Authors

Institutions
Kazuya Matsumoto' ${ }^{1}$ Masaru Ueki², Yohei Takeda', Kenichi Harada' ${ }^{1}$, Takumi Onoyama', Soichiro Kawata', Yuichiro lkebuchi ${ }^{1}$, Ryu Imamoto ${ }^{1}$, Yasushi Horie ${ }^{3}$, Yoshikazu Murawaki ${ }^{1}$

${ }^{1}$ Department of Gastroenterology, Tottori University Hospital, Yonago, Japan

${ }^{2}$ Center for Promoting Next-Generation Highly Advanced Medicine, Tottori University Hospital, Yonago, Japan

${ }^{3}$ Department of Pathology, Tottori University Hospital, Yonago, Japan

\section{Bibliography}

DOI http://dx.doi.org/

$10.1055 / \mathrm{s}-0034-1393076$

Published online: 23.9.2015

Endoscopy International Open

2015; 03: E662-E664

(c) Georg Thieme Verlag KG

Stuttgart · New York

E-ISSN 2196-9736

\section{Corresponding author \\ Masaru Ueki}

Center for Promoting Next-

Generation Highly Advanced

Medicine

Tottori University Hospital

36-1 Nishi-cho

Yonago 683-8504

Japan

Phone: +81-859-38-6745

Fax: +81-859-38-6745

ueki@med.tottori-u.ac.jp

\section{License terms}

()(1) $\Theta \circledast$
Background and study aims: Specimens collected by fine needle are microscopic and contain blood; therefore, the presence of a target specimen within a sample is often difficult to confirm. Although rapid on-site evaluation (ROSE) during endoscopic ultrasound-guided fine needle aspiration biopsy (EUS-FNA) is beneficial, many health care facilities are unable to apply this technique due to a lack of cytopathologists.

The aim of this study was to develop and validate a device that detects the target specimen within pancreatic tumor EUS-FNA samples.

Patients and methods: Fifty-eight consecutive patients with solid pancreatic masses were studied for a preliminary case series at a tertiary-care uni-

\section{Introduction}

$\nabla$

Endoscopic ultrasound-guided fine needle aspiration biopsy (EUS-FNA) has enhanced the diagnostic capabilities of endoscopic ultrasonography (EUS) by providing additional pathologic findings [1]. However, confirming the presence of a target specimen within a sample is often difficult because specimens collected with these fine needles are microscopic and contain blood. Rapid on-site evaluation (ROSE) during EUS-FNA is beneficial, but many health care facilities are unable to provide this service due to a lack of cytopathologists. Because fine-gauge needles are associated with fewer complications, easier to use than thicker needles, and equally effective for diagnosis, 25-G needles are commonly used for EUS-FNA of pancreatic tumors [2]. Given the tendency to perform EUS-FNA using fine-gauge needles, evaluation of the target specimen in EUS-FNA samples of pancreatic lesions, therefore, may be even more difficult.

To identify the wavelength that provided the optimal absorption specifically by blood covering the target specimen, single wavelengths from $435 \mathrm{~nm}$ to $695 \mathrm{~nm}$ were projected by light-emit- versity hospital (Tottori University Hospital, Yonago, Japan). The material collected was checked with a target sample check illuminator (TSCI) and was evaluated by one cytopathologist.

Results: The agreement rate between the TSCI and histopathology was $93.7 \%$. Further testing procedures were not needed in $91.4 \%$ of patients, and the mean number of needle punctures was 1.2 after a single pass using TSCI. No adverse events were encountered with the procedure.

Conclusions: With the introduction of the TSCI in EUS-FNA, it became possible to both collect the minimum necessary target samples by EUS-FNA and to end further procedures, even without performing ROSE.

ting diodes onto EUS-FNA samples of dog pancreas. We found that we were able to confirm the target specimen most clearly with observation using the 605-nm wavelength. We then developed a device equipped with a 605-nm wavelength light source, referred to as the target sample check illuminator (TSCI) ( $\bullet$ Fig. 1).

The current study verified the potential usefulness of this device, both in collecting the minimum necessary target specimens within EUSFNA samples of pancreatic tumors, and in eliminating the need for further procedures, even without performing ROSE.

\section{Patients and methods}

\section{$\nabla$}

Fifty-eight consecutive patients (33 men, 25 women; mean age 70.4 years; range, 42-87 years) were included in this prospective study ( Table 1), which took place from July 2012 to October 2013.The research protocol was reviewed and approved by the institutional review board of Tottori University Hospital.

Samples of EUS-FNA were observed using the TSCI prior to histologic diagnosis to determine 
Table 1 Details for EUS-FNA.

\begin{tabular}{|l|l|}
\hline & No. of patients \\
\hline Pancreatic masses & Adenocarcinoma (41) \\
\hline & Chronic pancreatitis (9) \\
\hline & Neuroendocrine tumor (3) \\
\hline & Metastatic tumor (3) \\
\hline & Accessory spleen (2) \\
\hline Benign/ Malignant & Benign (14) \\
\hline Needles & Malignant (44) \\
\hline & 19G (1) \\
\hline & 22G (26) \\
\hline Location (head/ body/ tail) & 25G (31) \\
\hline
\end{tabular}

Table 2 Utility of TSCI for EUS-FNA.

\begin{tabular}{ll} 
Agreement rate between $\mathrm{TSCl}$ and histopathology & $93.7 \%(133 / 142)$ \\
\hline $\begin{array}{l}\text { Rate of adequate cellularity obtained with a single } \\
\text { pass by a TSCI }\end{array}$ & $91.4 \%(53 / 58)$ \\
\end{tabular}

whether the presence of target specimens could be confirmed. EUS-FNA was performed using a 7.5-MHz, convex linear array echoendoscope (GF-UCT260; Olympus Optical, Tokyo, Japan) and a 19-G (M00550040; Boston Scientific Corporation, Natick, MA), 22-G (NA-200H-8022; Olympus Optical), or 25-G needle (M00550020; Boston Scientific Corporation).

Patients were referred for EUS-FNA based on the need to perform evaluations for any malignancies. Informed consent was obtained from all patients prior to EUS-FNA.
No algorithm was used to decide whether to use a 19-G, 22-G, or $25-G$ needle. Needles were selected based on the judgment of the attending physicians.

The aspirated material was evaluated by a cytopathologist, and ROSE was not used. The final diagnosis was verified histologically with either subsequent surgery or treatment based on the clinical course. Patients without a malignant disease, including those with chronic pancreatitis, a neuroendocrine tumor, or an accessory spleen, were followed up with imaging examinations.

All patients were closely observed for any immediate or delayed complications.

The data recorded include sample adequacy, final diagnosis, agreement rate between the TSCI and histopathology, and procedure-related complications.

\section{Results}

In the examinations, areas that included target specimens appeared orange (yellow arrows), and those without appeared dark brown (blue arrows) when examined with the TSCI ( $\bullet$ Fig. 2a). Examinations with a TSCI were performed when we were unable to detect target specimens with a white light ( $\mathbf{~ F i g . 2 b ) . ~}$ We were only able to detect target specimens using a white light in about 28 out of 57 cases, compared with 57 out of 58 cases when a TSCI was used $(P<0.001)$. The mean number of total needle punctures was 2.4 (range, $1-5$ ), and the agreement rate between TSCI and histopathology in 142 samples was 93.7\% (133/ 142). No differences were observed between cancerous and non-cancerous lesions in detection capacity. There were no differences between 19-G, 22-G and 25-G needles in detection capacity. When the presence of the target specimen was con-
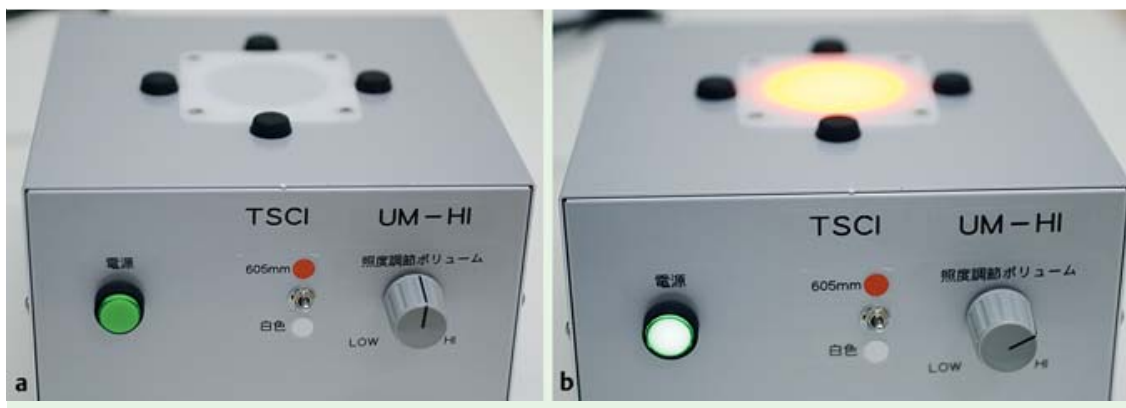

Fig. 1 We developed a device equipped with a 605-nm wavelength light source, referred to as the target sample check illuminator (TSCI).
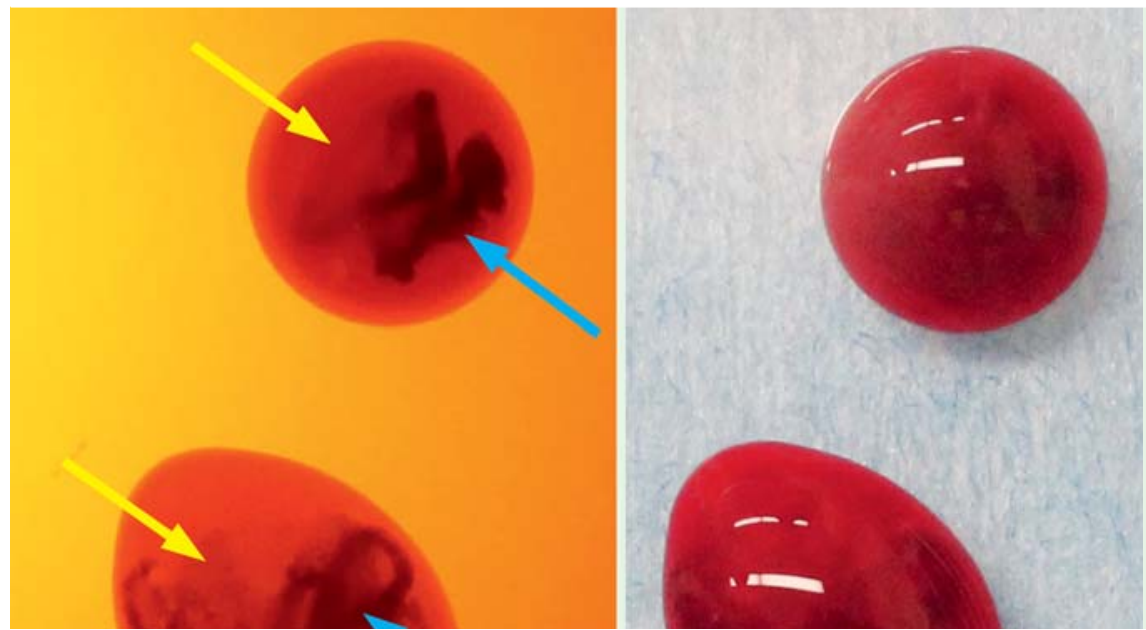

Fig. 2 a Areas with target specimens appeared orange (yellow arrows), and those without appeared dark brown (blue arrows) when observed with the TSCI. b We could not always detect target specimens in EUS-FNA samples under white light. 
Table 3 Diagnostic power of EUS-FNA when used with a TSCI*

\begin{tabular}{|lllllll}
$\mathbf{n}$ & Adequate specimen (\%) & Sensitivity (\%) & Specificity (\%) & PPV (\%) & NPV (\%) & Accuracy (\%) \\
\hline 58 & 98.3 & 97.7 & 100 & 100 & 93.3 & 98.3 \\
& $(57 / 58)$ & $(43 / 44)$ & $(14 / 14)$ & $(43 / 43)$ & $(14 / 15)$ & $(57 / 58)$
\end{tabular}

PPV, positive predictive value; NPV negative predictive value

* Materials for which a final diagnosis was obtained via surgery or clinical follow up.

firmed by TSCI, $91.4 \%$ (53/58) of patients were able to finish tests ( $\triangle$ Table 2 ). The percentage of cases that only required 1 EUS-FNA pass to yield a diagnosis was $84.5 \%(49 / 58)$, and the mean number of necessary needle punctures was 1.2 (67/58). No adverse events were encountered in the current study. An adequate specimen was obtained by EUS-FNA in $98.3 \%$ of cases. The sensitivity, specificity, positive predictive value, negative predictive value, and accuracy for EUS-FNA were $97.7 \%, 100 \%, 100 \%, 93.3 \%$, and $98.3 \%$, respectively ( $\odot$ Table 3 ). Only one case was misdiagnosed, a metastatic pancreatic carcinoma (renal cell carcinoma) that measured $15 \mathrm{~mm}$ and existed in the body of the pancreas. We could get only pancreas parenchyma; tumor cells were not included in the sample. Therefore, a difference in ability to diagnose due to the puncture site was not found.

\section{Discussion \\ $\nabla$}

This study verified the utility of the TSCI previously developed by us, which is based on the absorption spectrum of human oxyhemoglobin reported by Zijlstra et al [3]. When a target specimen was present in a sample observed with a light with a wavelength of $605 \mathrm{~nm}$, the blood appeared dark brown due to light absorption, and the target specimen appeared orange because it did not absorb the light.

The TSCI was designed to detect the presence of pancreatic tissue and not necessarily malignant specimens, thus a specimen considered adequate by a TSCI may not be representative of a tumor. However, the concordance rate between the TSCI and histopathologic examination of EUS-FNA samples was $93.7 \%$. Therefore, we were able to confirm that with the introduction of the TSCI in EUS-FNA, it was possible both to collect the minimum necessary target samples by EUS-FNA and to end further procedures, even without performing ROSE.
The difference between the mean number of total needle punctures and the mean number of necessary punctures was due to the need to maintain a high standards and accuracy for our patients. However, in the future, we will be able to finish sessions earlier based on evaluation of a TSCI. Specifically, by using a TSCI, results will be better than when using the standardized approach based on a minimum of two passes for all pancreatic masses.

The overall complication rate for EUS-FNA has been reported as $0 \%$ to $2 \%[1,4]$. We finished EUS-FNA with an average of 1.2 punctures using the TSCI, therefore, this approach seems likely to contribute to a decreased complication rate because many health care facilities are unable to perform ROSE due to lack of cytopathologists.

In conclusion, the introduction of use of a TSCI with EUS-FNA enabled both the collection of the minimum number of necessary samples by EUS-FNA and an end to further procedures, even without ROSE.

\section{Competing interest: None}

\section{References}

1 Hewitt MJ, McPhail MW, Possamai L et al. EUS-guided FNA for diagnosis of solid pancreatic neoplasm: a meta-analysis. Gastrointest Endosc 2012; 75: 319-331

2 Madhoun MF, Wani SB, Rastogi $A$ et al. The diagnostic accuracy of 22gauge and 25-gauge needles in endoscopic ultrasound-guided fine needle aspiration of solid pancreatic lesions: a meta-analysis. Endoscopy 2013; 45: 86-92

3 Zijlstra WG, Buursma A, Meeuwsen-van der Roest WP. Absorption spectra of human fetal and adult oxyhemoglobin, de-oxyhemoglobin, carboxyhemoglobin, and methemoglobin. Clin Chem 1991; 37: 16331638

4 Matsumoto K, Takeda Y, Harada $K$ et al. The effect of pancreatic juice cytology and/or endoscopic ultrasound-guided fine-needle aspiration biopsy for pancreatic tumor. J Gastroenterol Hepatol 2014; 29: 223 227 